\title{
Multilevel modeling of determinants of fertility status of married women in Ethiopia
}

\author{
Anteneh Mulugeta Eyasu \\ Department of Statistics, Arba Minch University, Arba Minch, Ethiopia
}

Email address:

antenehmulugeta6@gmail.com

\section{To cite this article:}

Anteneh Mulugeta Eyasu. Multilevel Modeling of Determinants of Fertility Status of Married Women in Ethiopia. American Journal of Theoretical and Applied Statistics. Vol. 4, No. 1, 2015, pp. 19-25. doi: 10.11648/j.ajtas.20150401.14

\begin{abstract}
The main objective of this study is to investigate the determinant factors of fertility status of married women in Ethiopia and to examine the reasons for variations of fertility across regions of Ethiopia based on data on 7052 married women obtained from Ethiopian Demographic and Health Survey (EDHS, 2011). Multilevel binary logistic regression models on fertility status of married women were employed. This study revealed that the random intercept and fixed slope model fits the data significantly better than the other multilevel logistic regression models. The results confirmed that woman's education level, sex of household head, being visited by family planning worker last twelve months, child loss experience, woman's occupation, religion and age of woman at first birth were found to be significant determinants and also contributing factors for variation in fertility status of married women among the regions of Ethiopia. In random intercept model the overall variance of constant term was found to be statistically significant implies that women with the same characteristics in two different regions have different fertility status: that is, there is a clear region effect. In this study multilevel model best fit the data as compared to single level model.
\end{abstract}

Keywords: DHS, High Fertility, Multilevel Logistic Analysis, Random Intercept Model

\section{Introduction}

Fertility is one of the three principal determinants of the size and structure of the population of a country (the other two being mortality and migration) [1]. High fertility defined as five or more births per woman over the reproductive career characterizes 33 countries of the world. Twenty-nine of these countries are in Sub-Saharan Africa. High fertility poses health risks for children and their mothers, detracts from human capital investment, slows economic growth, and exacerbates environmental threats [2]. Ethiopia is the secondlargest country in Africa, with an estimated population of nearly 79 million in 2010 and a growth rate of 2.6 percent per year. Ethiopia is a predominantly rural and young society, with 84 percent living mainly in densely populated highland settlements. While the urban population is growing at around 4 percent per year, the rural population is still growing at around 2.3 percent. The proportion of the population under age 15 is 45 percent, with only 3.2 percent above age 65 [3].

The World Bank has benchmarked a population growth rate of 2 percent per year as a level beyond which it is difficult for a country's institutions and technologies to keep up with expanding population pressures on all sectors, from water, sanitation, and agriculture to health, housing, and education. Ethiopia adds 2 million people every year, and it is the pace and imbalanced distribution of this population growth, rather than the ultimate size of the population, that most give rise to concerns. These concerns are aggravated by degradation of the environment and natural resources, increased climate variability, and market vulnerability [4].

High fertility is a major contributor to poverty. The addition of one more child increases the incidence of poverty. Reduction in fertility in rural areas is clearly a priority for the poverty reduction agenda [5]. High parity restricts women's educational and economic opportunities, thereby limiting their potential for empowerment broadly, as well as their ability to safeguard the health and economic well-being of the family and community at large. Low educational attainment further perpetuates high fertility, as these women tend to have less knowledge of and access to FP options [6]. In Ethiopia twenty-five percent of currently married women have an unmet need for family planning, 16 percent for 
spacing and 9 percent for limiting [7].

High fertility ranks second only to HIV/AIDS as the greatest perceived threat to individual and social well-being. High fertility also affects the well-being of mothers and their children. Maternal mortality and morbidity are strongly associated with high parity and early childbearing [8]. Significant declines in fertility have occurred in most regions of the world. In many sub-Saharan African countries the fertility transition remains in its early stages therefore fertility in Ethiopia remains the dominant factor dictating the future size, growth and composition of the population in the country. National level fertility declines also disguise significant variations within countries. Poor and rural populations often have the least access to family planning information and services, and thus the highest fertility rates [9]. Hence, the main objective of this study is to identify the determinant factors of fertility status and also to examine the causes and/or reasons for variations of fertility status across regions of Ethiopia.

\section{Methodology}

\subsection{Data}

In this study, data of the 2011 Ethiopia Demographic and Health Survey (EDHS) were used. A representative sample of 17,817 households was selected for the 2011 EDHS survey. Therefore, the analysis presented in this study is based on fertility data of 7,052 married women aged 15-49.

\subsection{Study Variables}

Dependent Variable: This study analyzed the specific measure of fertility; i.e. children ever born (CEB). CEB comprises information on the number of all children born up to the survey date. So the dependent variable used in this study is fertility status, which is categorized as low when $\mathrm{CEB}$ is less than five and high when CEB is greater or equal to five among currently married women [2].

Independent Variables: The explanatory variables to be studied as determinants of fertility status of married women are grouped into socio-economic, demographic and proximate factors.

Socioeconomic variables are woman's educational level, literacy, husband/partner's educational level, husband/partner occupation, religion, region and place of residence at the

$$
\log i t\left(P_{i j}\right)=\log \left(\frac{p_{i j}}{1-p i j}\right)=\beta_{0 j}+\beta_{1} x_{1 i j}+\beta_{2} x_{2 i j}+\ldots+\beta_{K} x_{k i j}, i=1, \cdots, n_{j}, j=1, \cdots, 11=\beta_{0 j}+\sum_{h=1}^{k} \beta_{h} x_{h i j}
$$

Where the intercept term $\beta_{0 j}$ is assumed to vary randomly and is given by the sum of an average intercept $\beta_{0}$ and group-dependent deviations $U_{0 j}$, that is

$$
\beta_{0 j}=\beta_{0}+U_{0 j}
$$

As a result we have: time of the survey, wealth index of household, mass media exposure, a woman's occupation, visited by family planning worker last twelve months and sex of household head.

Demographic variables are age of the woman at the time of the survey, child loss experience, age of woman at first birth.

Proximate variables are current contraceptive method use, knowledge of any contraceptive method and husband desire for children

\subsection{Multilevel Logistic Regression Model}

In this analysis, a multilevel statistical approach was used to model the relation between fertility status and the explanatory variables. Two levels of data hierarchy were stated (for instance individual women and region) in a multilevel logistic regression model. Units at one level are nested within units at the next higher level. In this study the basic data structure of the two-level logistic regression is a collection of $\mathrm{N}$ groups (regions) and within-group $\mathrm{j}$ $(j=1,2, \cdots, N)$, a random sample $n_{j}$ of level-one units (individual women). The response variable is denoted by $Y_{i j}$

$$
Y_{i j}= \begin{cases}1, & \text { if } i^{\text {th }} \text { woman having children ever born } \geq 5 \text { in } j^{\text {th }} \text { region } \\ 0, & \text { otherwise }\end{cases}
$$

With probabilities, $P_{i j}=P\left(Y_{i j}=1 \mid X_{i j}, U_{j}\right)$, is the probability of having children ever born greater than or equal to five for the $i^{\text {th }}$ married woman in the $j^{\text {th }}$ region and $1-P_{i j}=P\left(Y_{i j}=0 \mid X_{i j}\right)$ is the probability of having children ever born less than five for the $i^{\text {th }}$ married woman in the $j^{\text {th }}$ region.

\subsubsection{The Random Intercept Model}

The Random intercept model is used to model unobserved heterogeneity in the overall response by introducing random effects. In the random intercept model the intercept is the only random effect meaning that the groups differ with respect to the average value of the response variable, but the relation between explanatory and response variables cannot differ between groups.

The random intercept model expresses the log-odds, i.e. the logit of $P_{i j}$, as a sum of a linear function of the explanatory variables. That is,

$$
\log i t\left(P_{i j}\right)=\beta_{0}+\sum_{h=1}^{k} \beta_{h} x_{h i j}+U_{0 j}
$$

Where $\beta_{0}+\sum_{h=1}^{k} \beta_{h} X_{h i j}$ is the fixed part of the model. The remaining $U_{0 j}$ is called the random part of the model. It is assumed that the residual $U_{0 j}$ is mutually independent and 
normally distributed with mean zero and variance $\delta_{0}^{2}[10]$.

For this study parameter estimation for multilevel logistic model is done by Marginal Quasi Likelihood [11, 12]. The likelihood approach is used to estimate the fixed and the random parameters of the model through quadrature by treating the actual random effect $U$ as nuisance parameters, and work with the marginal likelihood function.

\section{Results of Multilevel Logistic Regression Analysis}

In the multilevel analysis, a two-level structure is used with regions as the second-level units and women as the first level units. This analysis is mainly aimed at a comparison among regions and within regions variation of fertility status of married women in Ethiopia. The hierarchical structure of the data is formed such that 7,052 individuals (women) were nested in eleven geographical regions based on the 2011 Ethiopia Demographic and Health survey data.

\subsection{Multilevel Logistic Regression Model Comparison}

The choice of relevant multilevel models is an important step, and it should be based on the necessity of parsimony in a model. Parsimony means that models should be as simple as possible [13]. To compare the fit of two models, calculate a $\chi^{2}$ statistic as the positive difference of 2 times their respective loglikelihoods. The positive difference of 2 times loglikelihood has a $\chi^{2}$ distribution with degrees of freedom obtained from the difference of the number of parameters to be estimated in the two models. If the statistic is not statistically significant, it suggests that a more complicated model may not be necessary [13]. And also given a set of candidate models for the data, the preferred model is the one with the minimum AIC and BIC value.

Hence, the deviance-based chi-square value for the empty model shown in Table 3.1 is the difference in log likelihoods between an empty model of single level logistic regression and empty model of multilevel logistic regression, which is to be compared with the critical value from the chi-squared distribution with 1 degree of freedom. The significance of this test

(Deviance $\left.\chi^{2}=201.69, d f=2-1=1, p=0.000<0.05\right)$ implies that an empty model with random intercept is better than an empty model without random intercept.

Table 3.1. Multilevel Logistic Regression Model Results for Fertility Status of Married Women with Their Deviance Based Chi-square Test Statistics and the Information Measures.

\begin{tabular}{llll}
\hline Fitted Model & Empty model & Random intercept model & Random coefficient model \\
\hline -2*log likelihood & 8617.47 & 6870.09 & 6864.06 \\
Deviance based chi-square test & 201.69 & 1747.38 & 6.033 \\
p-value & $0.000^{*}$ & $0.000^{*}$ & 0.303 \\
AIC & 8621.47 & 6920.09 & 6924.06 \\
BIC & 8635.12 & 7091.51 & 7129.89 \\
\hline
\end{tabular}

*significant at $5 \%$

The model comparison test of significant deviance based chi-square value, deviance $\chi^{2}=1747.381, d . f=25-2=23, p=0.000<0.05$ for random intercept model indicates that the random intercept and fixed slope model is a better fit as compared to the empty multilevel model. The deviance-based chi-square test, $\quad \chi^{2}=6.033, d . f=30-25=5, p=0.303$ of random effects for random coefficient model is not statistically significant at $5 \%$ level of significance. This implies that, as compared to the model with random intercept and fixed slope model, the random coefficient model is not a better fit. Thus, the deviance-based chi-square test presented in Table 3.1 shows that among multilevel logistic regression models, the random intercept and fixed slope model fits the data significantly better than the other multilevel logistic regression models. Similarly, the information measures, AIC and BIC, are also used to compare either nested or nonnested models. Thus, given a set of candidate models for the data, the preferred model is the one with the minimum AIC and BIC value. Thus, the AIC and BIC values shown on Table 3.1 also confirm that the random intercept and fixed slope logistic regression model fits the data well compared to the other multilevel models.

\subsection{Results of Empty Multilevel Logistic Regression Model}

In empty multilevel logistic regression model, the probability of having high fertility is only a function of the region in which the married woman live, which is accounted for with region level random intercept. This model contains no explanatory variables and it can be considered as a parametric version of assessing heterogeneity of fertility status of married women among regions of Ethiopia. Before analyzing data using the multilevel approach, there is a need to check for the heterogeneity of fertility status of married women with regard to regions.

The empty multilevel logistic regression results presented in Table 3.2 revealed that information in the fixed effects part, we can say that the estimated average log-odds of having high fertility for married women across regions is $\hat{\beta}_{0}=0.713$. We can convert this back to a probability 0.671 ; this is the overall proportion of women on high to low fertility of married women in Ethiopia without accounting for other sources of variation. The intercept for region $\mathrm{j}$ is $0.713+u_{0 j}$, where the variance of $u_{0 j}$, between regions in region average log-odds of having high fertility is estimated as $\hat{\delta}_{0}^{2}=0.464$. 
Table 3.2. Results for Multilevel Logistic Regression Model without Explanatory Variables.

\begin{tabular}{lllll}
\hline Fixed part & coefficients & S.E & Z-value & p-value \\
\hline$\beta_{0}$-int ercept & 0.713 & 0.142 & 5.01 & $0.000^{*}$ \\
Random part & Estimate & & & \\
Random intercept & 0.464 & 0.104 & 4.461 & $0.000^{*}$ \\
Rho & 0.061 & 0.026 & $201.69(\mathrm{LR})$ & $0.000^{*}$ \\
\hline
\end{tabular}

*significant at $1 \%$ level; S.E: standard error

The null hypothesis tested is $\delta_{0}^{2}=0$. i.e., there is no regional variation in married women experiencing fertility status in Ethiopia. Based on the results Wald $\chi^{2}=19.9=(Z-\text { value })^{2}=(0.464 / 0.104)^{2}, d f=1$ with the corresponding $\mathrm{p}$-value $=0.000$ less than 0.01 , the null hypothesis has to be rejected, indicating strong evidence that the between region variance is non zero. The variance of the random factor is significant which indicates that there are regional differences in experiencing fertility among married women and thus, multilevel analysis can be considered as an appropriate approach for further analysis than single level.

In order to get an idea of how much of variation in fertility status of married women is attributable to the region level factors, it is useful to see the intra-region correlation coefficient (ICC), which measures the proportion of variance of the high fertility of married women that is between regions, not within regions. The intra-region correlation (ICC) in intercept only model is 0.061 which is significant at $1 \%$ level of significance (Table 3.2). This means that around 6 percent of the variance in high fertility status among married women is due to differences across (between) regions, with the remaining 94 percent attributable to individual level, i.e., within region differences.

\subsection{Result of Random Intercept Multilevel Logistic Regression Model}

The probability of having high fertility is a function of the region in which the married women live, which is accounted for with a region level random intercept and of the individual variables. The intercept estimation is random at the regional level, $\operatorname{var}\left(u_{0 j}\right)$. Thus, the value of $\operatorname{var}\left(u_{0 j}\right)=\delta^{2}{ }_{0}=0.184$ is the estimated variance component of the intercept.

The multilevel logistic regression analysis result displayed in Table 3.3 confirmed the significance of regional difference in fertility status of married women in Ethiopia. The deviance based chi-square $=1747.381$, d. $f=23$, $p$-value $=0.000<0.05$ for random effects in random intercept model, suggesting that women with the same characteristics in two different regions have different fertility status in Ethiopia: that is, there is a clear regional effect.

The multilevel analysis also revealed that woman's educational level, sex of household head, visited by family planning worker twelve months before the survey, child loss experience, woman's occupation, religion and age of woman at first birth are significant determinants for fertility status and also contributing factors for variation in fertility status of married women among the regions of Ethiopia.

Table 3.3. Results of Random Intercept and Fixed Coefficient Logistic Regression Model.

\begin{tabular}{|c|c|c|c|c|c|c|c|}
\hline \multicolumn{8}{|l|}{ Fixed part } \\
\hline \multirow{2}{*}{ Fixed effect } & \multirow{2}{*}{$\hat{\beta}$} & \multirow{2}{*}{ S.E } & \multirow{2}{*}{$\mathbf{Z}$} & \multirow{2}{*}{ P-value } & \multirow{2}{*}{ Odds } & \multicolumn{2}{|c|}{ 95\% C.I for $\exp (\hat{\beta})$} \\
\hline & & & & & & lower & upper \\
\hline \multicolumn{8}{|c|}{ Woman's education (Higher education(ref.cat)) } \\
\hline No education & 2.047 & 0.206 & 9.95 & $0.000 *$ & 7.75 & 1.64 & 2.45 \\
\hline Primary & 1.049 & 0.201 & 5.23 & $0.000^{*}$ & 2.85 & 0.66 & 1.44 \\
\hline Secondary & 0.477 & 0.225 & 2.12 & $0.034 *$ & 1.61 & 0.04 & 0.92 \\
\hline \multicolumn{8}{|c|}{ Sex of household head (Male(ref.cat)) } \\
\hline Female & -0.361 & 0.0868 & -4.16 & $0.000^{*}$ & 0.7 & -0.5 & -0.2 \\
\hline \multicolumn{8}{|c|}{ Visited by family planning worker last twelve months (Yes(ref.cat)) } \\
\hline No & 0.408 & 0.078 & 5.21 & $0.000 *$ & 1.5 & 0.25 & 0.56 \\
\hline \multicolumn{8}{|c|}{ Child loss experience (None(ref.cat)) } \\
\hline At least one child & 2.15 & 0.082 & 26.21 & $0.000^{*}$ & 8.59 & 1.99 & 2.31 \\
\hline \multicolumn{8}{|c|}{ Woman's occupation (Not working(ref.cat)) } \\
\hline Agricultural wor. & 0.164 & 0.08 & 1.93 & 0.054 & 1.17 & 0 & 0.33 \\
\hline Non agricultural & 0.199 & 0.08 & 2.26 & $0.024 *$ & 1.21 & 0.03 & 0.37 \\
\hline \multicolumn{8}{|l|}{ Religion (Muslim (ref.cat)) } \\
\hline Orthodox & -0.117 & 0.08 & -1.38 & 0.169 & 0.88 & -0.3 & 0.05 \\
\hline Catholic & 0.669 & 0.33 & 1.99 & $0.047 *$ & 1.95 & 0.01 & 1.33 \\
\hline Protestant & 0.254 & 0.1 & 2.45 & $0.014 *$ & 1.28 & 0.05 & 0.46 \\
\hline Other & -0.097 & 0.25 & -0.38 & 0.707 & 0.9 & -0.6 & 0.41 \\
\hline Age of woman at first birth & -0.066 & 0.022 & -3 & $0.003 *$ & 0.93 & -0.11 & -0.023 \\
\hline Constant & -0.687 & 0.4 & -1.68 & 0.093 & & & \\
\hline Random part & Estimate & & & & & & \\
\hline Random intercept & 0.184 & 0.06 & 3.345 & $0.0004 *$ & & & \\
\hline Intra- region correlation(ICC) & 0.01 & 0.01 & 15.2 & $0.000^{*}$ & & & \\
\hline Deviance based chi-square & 1747.381 & & & $0.000^{*}$ & & & \\
\hline
\end{tabular}

${ }^{*} \mathrm{p} \leq 0.05 ;$ ref.cat $=$ reference category; S.E: standard error; C.I: confidence interval 
The multilevel logistic analyses indicate that woman's educational level is significantly associated with fertility status of married women at 5\% level of significance. The odds of having high fertility of married women who had no or primary or secondary education were significantly differing from those who had tertiary education. A woman who had no education is 7.748 times more (OR: 7.748, 95\% CI: $(1.643,2.45))$ likely to have high fertility than a woman who had tertiary education. In addition to this, the result showed that women who had primary education are 2.854 times more likely [OR: $2.854,95 \% \mathrm{CI}:(0.655,1.442)]$ to have high fertility than those who had tertiary education. Women who had secondary education are $61.1 \%$ more likely [1.611, 95\% CI: $(0.035,0.918)]$ to have high fertility than those who had tertiary education. This implies that women with the higher level of education (tertiary education) have the lowest chance of having high fertility (five or more children) compared to women who have no or low levels of education.

Another finding of this study indicates that sex of household head is significantly associated with fertility status of married women at $1 \%$ level of significance. Particularly, women from female headed family were $30.4 \%$ less likely to have high fertility than those from male headed family ([OR: $0.696,95 \% \mathrm{CI}:(-0.531,-0.19)])$. In this study multilevel logistic regression analysis result also showed that the experience of being visited by family planning worker twelve months before the survey is significantly associated with fertility status of married woman at $1 \%$ level of significance. Particularly, a woman who was not visited by family planning worker twelve months before the survey is $50.3 \%$ more likely to have high fertility than those woman visited by family planning worker during the same time ([OR:1.503, 95\% CI: $(0.254$, $0.561)]$.

This study also revealed that the likelihood of having high fertility for married women who had at least one child loss experience is 8.585 times more ([OR: $8.585,95 \% \mathrm{CI}$ : (1.989, 2.31)]) than those who had no child loss experience. Moreover, woman's occupation is significantly associated with fertility status of married women at 5\% level of significance. The odds of having high fertility for married women engaged in any activity (non agricultural) were significantly different from women who have no job. Women who are engaged in non agricultural [OR: $1.219,95 \% \mathrm{CI}$ : $(0.026,0.371)]$ sectors were more likely to have high fertility than those who have no job. The results of this study also indicate that religion is significantly associated with fertility status of married woman. The results indicate that age of married woman at first birth was found to be significantly associated with fertility status. Hence when age of a married woman at first birth is raised by one unit (one year) then the woman is $6.5 \%$ less likely to belong to high fertility group. Therefore, with other variables held constant, the odds of having high fertility (five or more children) decreases as age at first birth increases.

The fundamental reason for applying special statistical techniques in multilevel analysis is the likely existence of intra-class (intra-region) correlation arising from similarity of fertility status of married women of the same region compared to those of different regions of Ethiopia. Women having fertility status on the same region may be more similar to each other than women having fertility status in other regions, as they share a number of socioeconomic, social, cultural and other characteristics that may condition similar fertility status beyond what can be adjusted for by women level covariates. The results displayed in Table 3.3 showed that the intra-region correlation coefficient (ICC) is estimated as $\hat{\rho}=0.01$, the likelihood ratio test is 15.20 , pvalue $=0.00$ is less than 0.05 . This implies that ICC statistically significant at the $5 \%$ level of significance. This means that 1 percent of the total variability in fertility status of married women is due to differences across regions, with the remaining 99 percent attributable to individual differences.

\section{Discussion of Results}

This study examined the application and interpretation of multilevel logistic regression for explaining across region and within region heterogeneity in determining fertility status of married women in Ethiopia based on [7] data set. Accordingly multilevel logistic regression models on fertility status of married women were employed. The results obtained are discussed as follows. The multilevel logistic regression analysis result confirmed that woman's educational level, sex of household head, visited by family planning worker last twelve months, child loss experience, woman's occupation, religion and age of woman at first birth were significant predictors of fertility status of married women in Ethiopia.

This study showed that woman's educational level was significantly associated with fertility status of married women. The study indicates that women who had no education were more likely to have high fertility than those who had higher education. In addition to this, Women who had primary or secondary education were more likely to have high fertility than those who had higher education. This implies that women with the highest level of education (tertiary education) have the lowest chance of having high fertility (five or more children) compared to women who have no or low levels of education. A similar finding was obtained by [14] in Awassa town: poor educational status was found to have significant association with risk of having 5 or more CEB. A similar finding was also obtained in Bangladesh by [15]: they showed that higher education of women has a significant negative impact on fertility. The study done by [16] in Nepal showed that illiterate women are more likely to have a higher number of children than literate women. They argued that higher educated women should be able to implement their fertility preferences including their higher degree of autonomy in reproductive decision making, better access and information about contraception as well as cooperation from their husbands.

Another interesting finding of this study was that sex of 
household head was significantly associated with fertility status of married women. Particularly, women from female headed family were less likely to have high fertility than those households who are male headed family. This finding is consistent with the study done in Nepal [16] showed that women from female-headed households had fewer children than women from male-headed households. The finding of the present study also revealed that the experience of being visited by family planning worker twelve months before the survey was significantly associated with fertility status of married women at 5\% level of significance. A woman who was not visited by family planning worker twelve months before the survey is more likely to have high fertility than a woman visited by family planning worker twelve months before the survey. This finding is not consistent with the study done in Nepal [17] indicated that women who had family planning worker visit has no significant effect on fertility.

Another interesting result in this study was that the likelihood of having high fertility for married women who had at least one child loss experience is higher than women who had no child loss experience. This finding is consistent with the study done by [16] which confirms that women who had a child-death experience were more likely to have a higher number of children than those who had no such experience. Other study done in Awassa town by [14] showed that history of child or infant death was found to have strong positive association with fertility. Woman's occupation was found to be significantly associated with fertility of married women. Since, women who are engaged in non agricultural sector are more likely to have high fertility than those who have no job. This result contradicts with the study done by [14] which indicated that mothers' occupation did not show any significant association with the risk of having five or more CEB. However, the result of another study done in Bahir dar town, Ethiopia is in agreement with this study: income of mothers was found to be positively related with fertility of mothers [18]. This study found that religion was significantly associated with fertility of married woman. This result is not in agreement with the study by [14]: mother's religion is not significantly associated with the risk of having five or more CEB.

Age of woman at first birth was also significantly associated with fertility of married women. Hence when age of married woman at first birth is raised by one year then the woman is less likely to belong to high fertility status. This finding is similar with study done in Ethiopia which revealed that a situation in which mothers with an earlier age at first birth is likely to end up in having many children [19]. Also this finding is consistent with a study done by [20] which showed that age at first birth has a significant bearing on the number of children ever born.

\section{Conclusions}

The study revealed that generally, socio economic, demographic and proximate variables have a significant effect on fertility status of married women in Ethiopia. Particularly, woman's educational level, sex of household head, visited by family planning worker last twelve months before the survey, child loss experience, woman's occupation, religion and age of woman at first birth were significantly associated with fertility status of married women and also can be considered as the major significant factors that explain most of the variations in fertility status of married women among the regions of Ethiopia.

The study revealed that women with the highest level of education (tertiary education) have the lowest chance of having high fertility (five or more children) compared to women who have no education. The result also indicates that women from female headed family were less likely to have high fertility than those from male headed households. The study also showed that a woman who was not visited by family planning worker twelve months before the survey is more likely to have high fertility than a woman who was visited by family planning worker twelve months before the survey. The results suggest that when age of a married woman at first birth is increased then she will be less likely to belong to high fertility status.

The finding of this study also showed that the likelihood of having high fertility for married women who had at least one child loss experience was higher than married women who had no child loss experience. The study also concluded that the likelihood of having high fertility for married women who are engaged in non agricultural sector is more likely to have high fertility than those who have no job.

In multilevel data analysis it was found that among the three multilevel logistic models the random intercept and fixed slope model fits the data significantly better than the other multilevel logistic regression models. In random intercept model the overall variance of constant term was found to be statistically significant implying the existence of differences in fertility status of married women among regions of Ethiopia. This suggests that women with the same characteristics in two different regions have different fertility status: that is, there is a clear region effect. Hence, multilevel model best fit the data as compared to single level model.

\section{Recommendations}

Based on the findings of the study the following recommendations are forwarded:

Special focus should be given not only to promote education for non educated women but also to increase the level of women's education. Reproductive health programs should focus to have more family planning worker to alleviate high fertility. And also programs should focus to increase community awareness the effect of having baby at early stage and high fertility. Reproductive health programs that focus on reduction of child mortality should be considered to reduce fertility. The topic of further research will be to investigate regional variation in fertility status of married women by considering the spatial distribution of individual characterstics by applying spatial models. 


\section{Acknowledgments}

This work would have not been possible without the support of Dr. Butte Gottu, and Mr. Cheru Astemegiorgis. I would like to thank also Arba Minch University and Hawassa university who is financially helped me.

\section{References}

[1] United Nations (1973). Use of Model Headship Rates, chapter VI in Methods of Projecting House Holds and Families.

[2] United Nations (2009). World Population Prospects: New York: Population Division, Department of Economic and Social Affairs, United Nations.

[3] CSA and UNFPA (2008), Ethiopia, Summary and Statistical Report of the 2007 Population \& Housing Census, Population Census Commission. Addis Ababa, Ethiopia: UNFPA.

[4] Ethiopia Ministry of Finance and Economic Development (2008), Population Department, Population and Development Ethiopia, no. 1.

[5] ILO (2003). Working Out of Poverty, Report of the DirectorGeneral, International Labour Conference, 91st Session, Geneva.

[6] Mekbib, Tekle-Ab, Y.G., Hiwot, and M., Fantahun (2002). Survey of Unsafe Abortion in Selected Health Facilities in Ethiopia (unpublished report).

[7] EDHS (2011). Preliminary and final Report. Addis Ababa, Ethiopia and Calverton, Maryland, USA.

[8] CSA and ORC Marco (2001). Ethiopia Demographic and Health Survey. Addis Ababa. Ethiopia and Calverton, Maryland, USA.

[9] United Nations Population Fund (2005). International Migration and the Millennium Development Goals. Selected Papers of the UNFPA Expert Group Meeting; Marrakesh, Morocco. New York: UNFPA.
[10] Snijders, T. A. B. and Roel J. Bosker (1999). An Introduction to Basic and Advanced Multilevel Modeling, $1^{\text {st }}$ ed. Department of Statistics, University of Poone.

[11] Goldstein, H. (1991). Nonlinear Multilevel Models with an Application to Discrete Response Data. Biometrika, Vol. 78: PP. 45-51.

[12] Goldstein, H. and Rabash, J. (1996). Improved Approximations for Multilevel Models with Binary Responses. Journal of Royal Statistics Society, Vol. 159, PP. 505- 513.

[13] Hox, J. (2002). Multilevel Analysis: Techniques and Applications. Mahwah, N.J: Lawrence Erlbaum.

[14] Samson G. and Mulugeta B. (2009). Level and Differentials of Fertility in Awassa Town, Southern Ethiopia. Afr J Reprod Health; Vol. 13[1]: PP. 93-112

[15] Islam, S. and Kamrun, M. (2009). Fertility Transition in Bangladesh: The Role of Education. Proc. Pakistan Acad. Sci. Vol. 46(4): PP. 195-201.

[16] Adhikari, R. (2010). Demographic, Socio-economic, and Cultural Factors Affecting Fertility Differentials in Nepal. BMC Pregnancy and Childbirth, Vol. 10: PP. 19.

[17] Adhikari, R., Kusol, S. and Prasartkul, P. (2009). Correlates of Unintended Pregnancy among Currently Pregnant Married Women in Nepal. BMC International Health and Human Rights, Vol. 9, PP. 17.

[18] Eshetu W. (1998). The influence of Selected Social and Demographic Factors on Fertility: The Case of Bahirdar Town. Ethiopian Journal of Development Research; Vol. 20(1): PP. 119.

[19] Tewodros A., Jemal H. and Dereje H. (2010). Determinants of Adolescent Fertility in Ethiopia. Ethiop. J. Health Dev.; Vol. 24(1): PP. 30-38

[20] Bekele B. (2011). Determinants of High Fertility Status among Married Women: A Case Control Study in Kersa District, Eastern Ethiopia. MPH Thesis. Haramaya University, Ethiopia. 